\title{
CONSUMO DE ANTI-INFLAMATÓRIOS ENTRE UNIVERSITÁRIOS: UM ALERTA PARA O USO RACIONAL
}

\author{
Daniel Sarmento Bezerral \\ Tânia Regina Ferreira Cavalcanti ${ }^{\text {Il }}$ \\ Danielle Serafim Pinto III \\ Waléria Bastos de Andrade Gomes Nogueira \\ André Ricardo Bezerra Bonzi
}

\begin{abstract}
RESUMO
Os anti-inflamatórios não-esteroides (AINES) são usados em diversos distúrbios imunológicos e inflamatórios. O seu emprego, entretanto, tem sido feito, na maioria das vezes, de forma abusiva, causando grandes impactos na sociedade, inclusive mortes. Nesse contexto, o presente estudo tem como objetivo evidenciar o perfil do consumo de anti-inflamatórios, antipiréticos e analgésicos entre estudantes de medicina de uma Faculdade da rede privada de João Pessoa-PB. Alerta-se para a necessidade do uso racional destes medicamentos. Trata-se de uma pesquisa de campo com caráter descritivo, de abordagem quantitativa. A pesquisa foi realizada com discentes do Curso de Medicina da Faculdade de Medicina Nova Esperança e contou com a participação de 42 alunos do primeiro e segundo períodos do referido curso. $O$ estudo contemplou um questionário estruturado, dividido em duas partes: Parte I - dados de identificação dos participantes e Parte II - dados referentes ao uso de anti-inflamatórios, analgésicos e antitérmicos. A pesquisa foi aprovada pelo Comitê de Ética e Pesquisa FACENE/FAMENE com enfoque no método quantitativo. Os dados foram analisados por meio de estatística descritiva, através de frequência absoluta (número) e relativa (percentual), utilizando parâmetros de estatística descritiva. Dentre os estudantes entrevistados, a grande maioria relatou utilizar ao menos 2 medicamentos ao mesmo tempo com certa frequência. Integraram a pesquisa os seguintes medicamentos: paracetamol, dipirona, ibuprofeno e ácido acetilsalicílico, sendo o mais consumido o paracetamol. Ademais, 32 estudantes revelaram utilizar medicamentos sem prescrição médica o que gera preocupação acerca dos fatores que possam ser melhorados para que haja uma utilização mais racional destes medicamentos.
\end{abstract}

PALAVRAS-CHAVE: Analgésicos. Antipiréticos. Estudantes de medicina.

Graduando do curso de Medicina da Faculdade de Medicina Nova Esperança- FAMENE. João Pessoa, Paraíba, Brasil. Autor correspondente: E-mail: sarmentomeddaniel@gmail.com. ORCID ID: 0000-0003-1430-4796

Fisioterapeuta, Doutora, Docente da Faculdade de Medicina Nova Esperança- FAMENE, João Pessoa, Paraíba, Brasil. ORCID ID: 0000-0003-3084-6720

Farmacêutica, Doutora, Docente da Faculdade de Enfermagem Nova Esperança- FACENE, III João Pessoa, Paraíba, Brasil. ORCID ID: 0000-0002-9961-7558

Enfermeira, Mestre, Docente do curso de Enfermagem da Faculdade Maurício de Nassau, João IV Pessoa, Paraíba, Brasil. ORCID ID: 0000-0002-5208-108X

Enfermeiro. Faculdade Maurício de Nassau, E-mail: bonzipbgmail.com, João Pessoa, Paraíba, Brasil. ORCID ID: /0000-0001-5323-4095 


\section{INTRODUÇÃO}

A dor é entendida como uma experiência sensorial e/ou emocional na presença de lesão tecidual potencial ou real, podendo classificar-se em aguda ou crônica. A dor aguda sinaliza fator fisiológico de defesa, enquanto que a dor crônica envolve mecanismo de adaptação, apresentando como fator causal mais comum os processos inflamatórios. Estes são resultado do desequilíbrio dos sistemas de defesa e reparo do organismo humano. Assim, quando eles falham tem-se a inflamação e, a partir daí, recorre-se ao tratamento farmacológico. $\mathrm{O}$ processo inflamatório começa com a liberação de mediadores como a histamina, que é derivada da histidina, através da ação da histidina descarboxilase. ${ }^{1,2}$

A histamina, assim como a acetilcolina (Ach), não foi descoberta a partir de sua ação farmacológica e sim pela observação empírica de seu comportamento. Por outro lado, as células do sistema de defesa, como os mastócitos e basófilos, são ricas em histamina e repletas de grânulos intracelulares que armazenam a substância. Ademais, a histamina é liberada por um aumento do íon cálcio no citosol celular e possui uma ação importante nos processos de úlcera péptica, já que ela aumenta a produção de suco gástrico no estômago. ${ }^{1}$ Por outro lado, os eicosanoides, em mamíferos, participam de vários processos e também atuam como mediadores inflamatórios, pois se ligam a superfície celular e ativam segundos mensageiros. 3

A Aspirina é o grande precursor de fármacos com padrão anti-inflamatório, antipirético e analgésico. Logo, medicamentos como dipirona, paracetamol e ibuprofeno por serem sintetizadas a partir da aspirina, são classificados como Anti-inflamatórios não-esteroides
VOLUME 17 - NÚMERO 2 - AGO/2019

ISSN ELETRÔNICO 2317-7160
(AINES). Estes, são usados em diversos distúrbios imunológicos e inflamatórios e foram introduzidos na prática médica em meados da década de 80 , proporcionando alívio para dores e edemas, artrites, entorses e traumas esportivos. Ademais, são prescritos para o pós-operatório, período menstrual e enxaquecas. Estão disponíveis para venda em diversas formulações como géis, comprimidos e injetáveis, apresentando, essencialmente, três finalidades terapêuticas, tais como anti-inflamatória, analgésica e antipirética. ${ }^{1}$

Os AINES mais conhecidos e comercializados são o paracetamol, a dipirona, o ácido acetilsalicílico e o ibuprofeno. Estes carregam efeitos colaterais bastante parecidos, como irritação da mucosa gástrica, comprometimento do fluxo sanguíneo renal, além de serem potencialmente causadores de trombos por inibição de prostaglandinas $\mathrm{PGl}_{2}$. Ademais, tem-se evidenciado que o emprego destes medicamentos tem sido feito de forma irracional, causando grandes impactos na sociedade, inclusive mortes. $^{1}$

A literatura digital é rica em estudos farmacêuticos e pobre acerca da utilização destes fármacos. Logo, novos estudos visando a observação e reflexão desta utilização, assim como do entendimento dos estudantes acerca dos riscos envolvidos, faz-se necessário. Nesse contexto, a presente pesquisa tem como objetivo evidenciar o perfil do consumo de anti-inflamatórios, antipiréticos e analgésicos entre estudantes de medicina de uma Faculdade da rede privada de João Pessoa-PB, alertando-se para a necessidade do uso racional desses medicamentos, a fim de causarem menor impacto na saúde dos usuários. 


\section{MATERIAL E MÉTODOS}

A pesquisa foi aprovada pelo Comitê de Ética e Pesquisa (CEP), apresentando como número CAAE 59464116.4.0000.5179. O presente estudo caracterizou-se como uma pesquisa de campo, com caráter descritivo, com abordagem quantitativa acerca do consumo de anti-inflamatórios não-esteroides (AINES), dentre eles paracetamol, dipirona, ibuprofeno e ácido acetilsalicílico. Foi realizada nas Faculdades Nova Esperança FACENE/FAMENE e contou com a participação de 42 discentes do primeiro e segundo períodos do curso de Medicina escolhidos ao acaso, os quais responderam a um questionário estruturado.

O questionário constituiu-se de duas partes: Parte I - dados de identificação dos par-

\section{RESULTADOS E DICUSSÃO}

Conforme representado na Tabela 1, os anti-inflamatórios (AINES) mais utilizados pelos estudantes são respectivamente: paracetamol (52,4\%), dipirona (38,1\%) e ibuprofeno (9,5\%). O paracetamol e a dipirona, usados isoladamente ou em associação, apresentam bom resultado terapêutico no manejo de cefaleia, febre e ticipantes como: sexo, idade e renda familiar. Parte II - dados referentes aos hábitos de consumo de anti-inflamatórios, analgésicos e antipiréticos, quando os estudantes foram questionados acerca de qual medicamento consumido com mais frequência, dentre o paracetamol, dipirona, ibuprofeno e ácido acetilsalicílico; do acometimento de reações alérgicas ou efeito colateral/adverso; do uso de um ou mais fármacos ao mesmo de tempo; e se a utilização destes medicamentos acontecia mediante prescrição médica.

Os dados foram analisados por meio de estatística descritiva através de frequência absoluta (número) e relativa (percentual). resfriado comum e são os mais utilizados pelos acadêmicos. Ainda que a dipirona seja um excelente analgésico e antitérmico, o paracetamol segue, tanto no Brasil quanto nos Estados Unidos, como o medicamento mais utilizado como primeira escolha para o tratamento de dores leves a moderadas. ${ }^{14}$

Tabela 1. Perfil do consumo de anti-inflamatórios não-esteroides (AINES) por estudantes do curso de Medicina de uma instituição de ensino particular $(n=42)$

\begin{tabular}{lcccc}
\hline \hline \multirow{2}{*}{ AINES } & & \multicolumn{3}{c}{ CONSUMO } \\
\cline { 1 - 3 } \cline { 4 - 5 } & & HOMENS & MULHERES & TOTAL \\
PARACETAMOL & 8 & 14 & $22(52,4 \%)$ \\
DIPIRONA & & & 8 & $16(38,1 \%)$ \\
IBUPROFENO & 1 & 3 & $4(9,5 \%)$ \\
ÁC. ACETILSSALICÍLICO & & 0 & 0 & $0(0 \%)$ \\
\hline \hline
\end{tabular}

* Pergunta: Qual o medicamento abaixo você usa com maior frequência? Ibuprofeno ( ) AAS ( ) Paracetamol ( ) Dipirona ( ); Fonte: própria pesquisa, 2016; Fonte: próprio autor, João Pessoa -PB, 2016 
No presente estudo, o paracetamol ainda é a primeira escolha entre as mulheres entrevistadas. É importante inferir que mesmo o ibuprofeno não aparecendo entre os mais consumidos é considerado pela indústria farmacêutica como o fármaco mais seguro quando em monoterapia para uso na pediatria. ${ }^{15}$

Ademais, a utilização de AINES como também de derivados do protótipo Ácido Acetilsalicílico estão fortemente ligados a problemas gástricos e renais, já que bloqueiam a ação de COX 1 e 2 (cicloxiganases), responsáveis por parte do metabolismo de proteção do organismo humano. Além disso, o aumento da dose não contribui para a eficácia terapêutica e pode resultar em aumento dos efeitos adversos. ${ }^{15}$

Quando os estudantes foram questionados acerca do uso associado dos AINES (paracetamol, dipirona, ibuprofeno e ácido acetilsalicílico), 17 relataram utilizar 2 desses medicamentos ao mesmo tempo com certa frequência, enquanto que 4 estudantes afirmaram fazer uso de 3 medicamentos (Tabela 2). Em estudo realizado no Rio Grande do Sul, com estudantes de enfermagem, acerca do autoconsumo de AINES, foi verificado que dos 45 participantes, $44 \%$ optam pelo uso paracetamol. ${ }^{16}$

Tabela 2. Consumo mútuo dos anti-inflamatórios não-esteroides (AINES), paracetamol, dipirona, ibuprofeno e ácido acetilsalicílico, por estudantes do curso de Medicina de uma instituição de ensino particular $(\mathrm{n}=42)$. João Pessoa, 2016

\begin{tabular}{|c|c|c|c|}
\hline \multirow{2}{*}{$\begin{array}{c}\text { AINES consumidos ao } \\
\text { mesmo tempo }\end{array}$} & \multicolumn{3}{|c|}{ CONSUMO } \\
\hline & HOMENS & MULHERES & TOTAL \\
\hline 2 & 5 & 12 & $17(40,47 \%)$ \\
\hline 3 & 2 & 2 & $4(9,53 \%)$ \\
\hline 4 & 0 & 0 & $0(0,0 \%)$ \\
\hline Não sei (mais de um) & 0 & 0 & $0(0 \%)$ \\
\hline
\end{tabular}

Ao menos 10 alunos utilizaram, frequentemente, algum dos medicamentos citados sem prescrição médica e, pelo menos, 17 responderam utilizar com pouca frequência. Relataram consumir os medicamentos sem receita e sem orientação médica, 8 estudantes. Por outro lado, 29 estudantes compraram AlNES sem receita (Tabela 3 ).

A automedicação é a iniciativa de usar um fármaco, no intuito de ser beneficiado, através de informações recebidas por terceiros ou, pela mídia, sem a indicação de um médico ou odontólogo. Esta prática é bastante recorrente dentro do âmbito familiar e social, uma vez que é fácil adquirir receitas com vizinhos e amigos. Sendo importante ressaltar o perigo desta prática já que negligencia a orientação dos profis- sionais adequados. ${ }^{17}$

$\mathrm{O}$ uso de medicamentos sem prescrição e/ou orientação médica gira entre 38,0\% a $97,8 \%$, de acordo com o país de origem dos estudantes, do curso de graduação e da cultura da população na qual estão inseridos.5,6 O fato de estudantes de Medicina que, eventualmente, originam-se de classes empoderadas financeiramente e educacionalmente, estarem se automedicando vai ao encontro do comportamento da população leiga e desinformada.

Este comportamento, além de irresponsável, gera uma ratificação, mesmo que vaga, acerca do uso sem orientação, o que é muito perigoso do ponto de vista da saúde da comunidade. 7,8 
TABELA 3: Hábito de consumo de anti-inflamatórios não-esteroides (AINES) por estudantes do curso de Medicina de uma instituição de ensino particular $(n=42)$. João Pessoa, 2016

\begin{tabular}{cccc}
\hline \hline \multirow{2}{*}{ *ompra os AINES } & \multicolumn{3}{c}{ CONSUMO } \\
\cline { 3 - 5 } \cline { 3 - 4 } & HOMENS & MULHERES & TOTAL \\
\hline Com consulta médica e com receita & 2 & 2 & $4(9,53 \%)$ \\
Sem consulta médica e sem receita & 5 & 3 & $8(19,05 \%)$ \\
Receita sem consulta médica & & 0 & $0(0,00 \%)$ \\
Compra sem receita & 11 & 6 & $29(69,05 \%)$ \\
\hline \hline **Hábito de consumo & 4 & 18 & $10(23,80 \%)$ \\
\hline \hline Com muita frequência & 7 & 9 & $17(40,47 \%)$ \\
Com pouca frequência & 7 & 0 & $16(38,09 \%)$ \\
Uso raro & 0 & $0 \%(0 \%)$ \\
Não sabe & & 0 & 0 \\
\hline
\end{tabular}

*Pergunta: Quando você utiliza algum dos medicamentos, paracetamol, dipirona, ibuprofeno e ácido acetilsalicílico, você: ( ) sempre faz consulta médica e pega receita; ( ) nunca faz consulta médica e nunca pega receita; ( ) pega a receita sem consulta médica; ( ) compra a medicação na farmácia sem receita; **Pergunta: Qual o seu hábito de consumo para estes medicamentos? ( ) uso com muita frequência; ( ) uso com pouca frequência; ( ) uso raramente; ( ) nunca usei; Fonte: próprio autor, João Pessoa -PB, 2016

Em estudo semelhante, realizado entre 145 universitários, observou-se que a automedicação dos estudantes pode vir a gerar consequências negativas independentemente da doença, sintoma ou medicamento utilizado. Dos 145 entrevistados, 113 utilizaram algum tipo de anti-inflamatório não esteroidal, sem prescrição médica, alegando a facilidade na compra e o objetivo de alívio rápido para os sintomas. ${ }^{2,5}$

Embora nenhum dos estudantes tenha relatado problemas com o uso de AINES, faz-se necessário um controle desse consumo, já que podem trazer aumento nos eventos

\section{CONSIDERAÇÕES FINAIS}

Neste estudo, foi constatado que alunos de curso de Medicina de uma instituição de ensino particular têm o hábito de consumir anti-inflamatórios sem prescrição e orientação médica. Aproximadamente $60 \%$ dos alunos entrevistados fazem uso desse tipo de medicamento de pouca a muita frequência. 19\% fazem uso sem orientação médica, enquanto que $69 \%$ afirmam comprá-los sem receita. tromboembólicos, além do uso crônico e indiscriminado diminuir a proteção gástrica e renal. 9,10,11,12,13,17

É fácil evidenciar que a propaganda, a indicação por terceiros e a falta de controle dos órgãos de vigilância acerca dos anti-inflamatórios, facilita e incentiva o consumo destes fármacos sem orientação e prescrição médica. Ao mesmo tempo, estudantes de graduação da área de saúde, especificamente medicina, têm acesso a dados técnicos que criam a falsa sensação de segurança para o uso destes medicamentos. 


\title{
CONSUMPTION OF ANTI-INFLAMMATORY BETWEEN UNIVERSITY: AN ALERT FOR RATIONAL USE
}

\begin{abstract}
Non-steroidal anti-inflammatory drugs (NSAIDs) are used in a variety of immune and inflammatory disorders. Its use, however, has been done, most of the time, in an abusive way, causing great impacts on society, including deaths. In this context, the present study aims to highlight the profile of anti-inflammatory, antipyretic and analgesic consumption among medical students of a Private School in João Pessoa-PB, alerting to the need for rational use of these drugs. It is a field research with a descriptive character and a quantitative approach. The research was carried out with students of the medical school of the Nova Esperança College and had the participation of 42 students of the first and second periods of said course. The study included a structured questionnaire, divided into two parts: Part I - identification data of the participants and Part II - data regarding the use of anti-inflammatories, analgesics and antipyretics. The research was approved by FACENE / FAMENE Ethics and Research Committee with focus on the quantitative method. Data were tabulated using the SPSS statistical package (Version 18), using descriptive statistics parameters. Among the students interviewed the vast majority reported using at least 2 medications at the same time with a certain frequency. The following drugs were included in the research: Paracetamol, Dipyrone, Ibuprofen and Acetylsalicylic Acid, paracetamol being the most consumed. In addition, 32 students have reported using non-prescription medicines, which raises concerns about factors that can be improved for a more rational use of these drugs.
\end{abstract}

KEYWORDS: Analgesics. Antipyretics. Consumption. Medical students.

\section{REFERÊNCIAS}

1. Ritter J, Graeme RF, Rang HH. Farmacologia. 6 ed. Rio de Janeiro: Elsevier; 2007.

2. Martinez JE, Pereirab GAF, Ribeirob LGM, Nunesb R, Iliasb D, Navarrob LGM. Estudo da automedicação para dor musculoesquelética entre estudantes dos cursos de enfermagem e medicina da Pontifícia Universidade Católica - São Paulo. Rev. Bras. Reumatol. 2014 Mar; 54(2): 90-94.

3. Bruton LL, Chabner BA, Knollmann BC. As Bases Farmacológicas da Terapêutica de Goodman \& Gilman. 12. Ed. Porto Alegre: 2012.

4. Steinke JW, Negri J, Liu L, Payne SC, Borhs L. Aspirin Activation of Eosinophils and Mast Cells: Implications in the Pathogenesis of Aspirin-Exa- cerbated Respiratory Disease. 2014 Jul; 193(1): 41-47.

5. Muria S, Secoli GSR. Automedicação em estudantes de enfermagem do Estado do Amazonas - Brasil. Rev. Gaúcha Enferm, Porto Alegre. 2017 Marc; 38(1): 1-7.

6. Arrais PSD, Fernandes MEP, Pizzol TS, Ramos LR, Mengue SS, Luiza VL, et al. Prevalence of self-medication in Brazil and associeted factors. Rev Saúde Pública, 2016 Dez: 50(2): 1-11.

7. Rasheed F, Naqvi AA, Ahmad R, Ahmad N. Academic Stress and Prevalence of Stress-Related Self-Medication among Undergraduate Female Students of Health and Non-Health 
Cluster Colleges of a Public Sector University in Dammam, Saudi Arabia. J Pharm Bioallied Sci. 2017 Out; 9(4): 251-58.

8. Okyay RA, Erdoğan A. Self-medication practices and rational drug use habits among university students: a cross-sectional study from Kahramanmaraş, Peer J. 2017.

9. Ely LS, Engroff P, Guiselli SR, Cardoso GC, Morrone FB, Carli G. A. Uso de anti-inflamatórios e analgésicos por uma população de idosos atendida na Estratégia Saúde da Família. Rev. bras. geriatr. Gerontol. $2015 \mathrm{Jul}$; 18(3): 475-485.

10. Batlouni M. Anti-inflamatórios não esteroides: Efeitos cardiovasculares, cérebro-vasculares e renais. Arq. Bras. Cardiol. 2010 Apr; 94(4): 556-563.

11. Carli DM, Pires RC, Rohde SL, Kavalco CM, Fagundes RB. Peptic ulcer frequency differences related to h. Pylori or aines. Arq. Gastroenterol. 2015 Jan; 52(1): 46-49.

12. Shusuke H, Hiroyuki Y, Tatsuo T, Kazuki Y, Naoya K, Takeshi M, et al. Acetaminophen administration and the risk of acute kidney injury: a self-controlled case series study. Clin Epidemiol. 2018 Mar; 10: 265-276.

13. Sriuttha $P$, Sirichanchuen B, Permsuwan U. Hepatotoxicity of Nonsteroidal Anti-Inflammatory Drugs: A Systematic Review of Randomized Controlled Trials. Int J Hepatol, 2018 Jan; 1-13.

14. Prado MAMB, Bergamo PMS, Bastos FTF, Barros MB. A. Uso de medicamentos prescritos e automedicação em homens. Rev. bras. Epidemiol. 2016 Jul; 19(3): 594-08.

15. Ferreira TR, Lopes LC. Análise do uso de drogas analgésicas, antipiréticas e não-inflamatórias em prescrições pediátricas. J. Pediatr. 2016 Jan; 92(1): 81-87.

16. Fábio GF, Janaina SMS, Roberta SPP. Prevalência da Automedicação em Acadêmicos de Enfermagem em uma Faculdade de Caxias do Sul. Revista Contexto \& Saúde. 2019 Jan; 19(36): 45-52.

17. Silvia RS, Erika AM, Sandra CF, Ligiana PC, Nicolina SRL. Tendência da prática de automedicação entre idosos brasileiros entre 2006 e 2010: Estudo SABE. Rev Bras Epidemiol. 2018; 21(2). 